\title{
Germanica
}

\section{Rückkehr und vergebliche Hoffnung}

Zu Peter Huchels Gedicht «Heimkehr»

Retour et espoir vain - A propos du poème de Huchel Heimkehr

\section{Ludwig Volker}

\section{CpenEdition}

\section{Journals}

Édition électronique

URL : http://journals.openedition.org/germanica/2809

DOI : 10.4000/germanica.2809

ISSN : 2107-0784

Éditeur

Université de Lille

Édition imprimée

Date de publication : 30 juin 1987

Pagination : 107-123

ISSN : 0984-2632

Référence électronique

Ludwig Volker, « Rückkehr und vergebliche Hoffnung », Germanica [Online], 1 | 1987, Online erschienen am: 19 November 2015, abgerufen am 06 Oktober 2020. URL : http://journals.openedition.org/ germanica/2809; DOI : https://doi.org/10.4000/germanica.2809

Ce document a été généré automatiquement le 6 octobre 2020.

(c) Tous droits réservés 


\title{
Rückkehr und vergebliche Hoffnung
}

\author{
Zu Peter Huchels Gedicht «Heimkehr» \\ Retour et espoir vain - A propos du poème de Huchel Heimkehr
}

\author{
Ludwig Volker
}

\section{I}

1 Der Verlust eines naiven Heimatgefühls ist eine Grunderfahrung modernen Bewußtseins. Die Rückkehr in die Heimat wird nicht mehr fraglos als die Heimkehr in einen unberührten Lebensund Glücksraum empfunden - zu mächtig sind die Störungen geworden, die diesen Raum bedrohen, und sie kommen keineswegs nur von außen, vielmehr haben sie ihre eigentliche Quelle im Ich selbst. Von Hölderlins zweifelnder Frage:

Ihr teuern Ufer, die mich erzogen einst,

Stillt ihr der Liebe Leiden, versprecht ihr mir,

Ihr Wälder meiner Jugend, wenn ich

Komme, die Ruhe noch einmal wieder? ${ }^{1}$

2 bis zum zögernden Innehalten von Kafkas Heimkehrer-Gestalt vor dem Öffnen der Küchentür ${ }^{2}$ spitzt sich die literarische Erfahrung der Heimkehr auf die Frage zu, ob die Heimkehr nicht deshalb mißlingt, mißlingen muß, weil das Ich zur Heimkehr unfähig ist, weil es, wie der heimkehrende Sohn bei Kafka, «sein Geheimnis wahren will».

Die Literatur des 20. Jahrhunderts kennt viele Beispiele solchen Fremdwerdens nicht in der Fremde, sondern in der Heimat, im Schoß der Familie, im eigenen Lebens- und IchBezug. Andererseits erfährt der Heimat-Begriff im 20. Jahrhundert durch die gesellschaftlichen und geschichtlichen Veränderungen eine neue Konkretisierung: gegenüber der massiven Entfremdung, der der Einzelne in seiner alltäglichen Lebenspraxis ausgesetzt ist, oder gar jener vernichtenden Entfremdung, welche zwei Weltkriege über ihn gebracht haben, gewinnt der Begriff «Heimat» eine fast verlorengegangene Unmittelbarkeit zurück. Daß auch dieser konkrete Heimat-Begriff nicht frei von Störungen ist - so ist die Heimkehr aus dem Krieg in eine vermeintlich friedliche Heimat nur zu oft eine Illusion und der angebliche private Freiraum nicht 
gefeit gegen die öffentlich-gesellschaftliche Überfremdung -, ändert nichts an der Tatsache, daß der Begriff Heimat in der Literatur des 20. Jahrhunderts in zwei Bedeutungsrichtungen auftritt: einer konkret-materiellen und einer geistigexistentiellen. Beide Bedeutungen ergänzen und relativieren sich gegenseitig: angesichts der tödlichen Bedrohung des natürlichen Lebensraums verkommt die literarische Gestaltung existentieller Fremdheit leicht zum ästhetizistischen Klischee, wie andererseits die Sorge um die Erhaltung des Lebensraums in die Gefahr gerät, die kompliziert gewordene Bewußtseinsstruktur des modernen Ichs zu nivellieren.

Am ausgeprägtesten findet sich der zweite, konkrete Heimat-Begriff, sieht man von neueren Thematisierungen eines ökologischen Bewußtseins ab, in literarischen Werken, die die Heimkehr aus dem Krieg zum Thema haben. Die Lyrik zeichnet sich hierbei gegenüber dem Drama (vgl. etwa Wolfgang Borcherts «Draußen vor der Tür», 1947) und dem Roman (vgl. Alfred Döblins «Hamlet oder Die lange Nacht nimmt ein Ende», 1946) dadurch aus, daß sie den Vorgang der Heimkehr aus der begrenzten Perspektive eines subjektiven Erlebens darstellt. Das heißt nicht, daß im lyrischen Gedicht Erfahrung und künstlerische Gestaltung eins seien und das Gedicht nur widerspiegele, was als Erlebnisinhalt schon vorgegeben ist. Im Gegenteil: gerade die lyrischen Gestaltungen des Heimkehr-Themas machen deutlich, daß die Erfahrung entsprechend der Art und Weise, wie sie ästhetisch objektiviert wird, eine Form annimmt, die deutliche Züge des Stilisierten, d.h. der bewußten subjektiven Sinngebung und Selbstinterpretation verrät. Hans Bender hat im Kommentar zu einem eigenen Heimkehr-Gedicht das Auseinanderfallen von Erfahrung und Ausdrucksform hervorgehoben:

Viele Tage später schrieb ich mein Gedicht. Enthielt es tatsächlich die erlebte Heimkehr? Oder die Heimkehr, über die ich in den Romanen und in den Gedichten anderer gelesen hatte? Oder war es jene Heimkehr, die ich mir in den Jahren der Gefangenschaft imaginiert hatte? ${ }^{3}$

5 Heimkehr-Gedichte sind demnach nur bedingt als authentische zeitgeschichtliche Dokumente anzusehen, vielmehr spiegelt sich in ihnen ein komplexer individueller Erfahrungshorizont, vor dem das geschichtliche Erleben und die produktive geistige Deutung und Aneignung des Erlebten sich durchdringen. Was einer erlebt hat, wird so in gewissem Sinne davon bestimmt, was er selbst als sein Erleben ästhetisch definiert. $\mathrm{Da}$ dabei Interpretations-muster eine Rolle spielen, die - Benders Selbstkommentar weist darauf hin - auf Gelesenes, auf Inhalte der Kultur, der der Einzelne angehört, zurückgehen, liegt auf der Hand. Einige Beispiele aus deutschen Heimkehr-Gedichten nach 1945 mögen die Spannweite der hierbei wirksamen Vorstellungen belegen: - aus einer Perspektive der Dankbarkeit über das gerettete Leben schreibt Becher sein Gedicht «Heimgekehrt» mit den Versen:

Er möchte knien und auf den Knien

Für seine Heimkehr danken ${ }^{4}$

- hingegen stellt Nelly Sachs in ihrem «Chor der Geretteten» den Begriff der Rettung selbst in Frage und weist auf die bleibenden Verletzungen hin, die der Krieg und das NS-Regime denen, die mit dem Leben davongekommen sind, zugefügt hat:

Wir Geretteten, Immer noch essen an uns die Würmer der Angst ${ }^{5}$

7 - das Gefühl, in ein anderes, als «Heimat» nicht wiederzuerkennendes Land zurückgekehrt zu sein, bestimmt die Darstellung bei Arnim T. Wegner: 
Ich kehrte heim, aber das Land, in dem ich gelebt hatte,

war nicht mehr $\mathrm{da}^{6}$

- naturgemäß dominiert in den meisten Heimkehr-Gedichten das Moment der Zerstörtung. So thematisieren Gedichte von Ricarda Huch und Marie-Luise Kaschnitz ${ }^{7}$ das Bild der vom Krieg verwüsteten Vaterstadt; hierher gehören auch Brechts Gedichte «Bericht des Sohnes» und «Rückkehr» mit seiner doppelten Perspektive aus der Sicht des Heimkehrenden («Die Vaterstadt, wie find ich sie doch?») und aus der Sicht der Vaterstadt («die Vaterstadt, wie empfängt sie mich wohl?») ${ }^{8}$ Manche HeimkehrGedichte indessen gehen über die Darstellung der Heimkehr-Situation hinaus und versuchen, dieser (und damit dem Kriegsgeschehen insgesamt) eine über den augenblicklichen Bezug hinausreichende Bedeutung zu geben:

9 - So stilisiert Elisabeth Langgässer die Heimkehr als Rückkehr aus dem Totenreich, wobei Odysseus, der klassische Heimkehrer der Weltliteratur, Pate steht:

Aus dem Reich der Kröte

Steige ich empor,

Unterm Lid noch Plutons Röte

Und des Totenführers Flöte

Gräßlich noch im Ohr ${ }^{9}$

10 - In den Horizont einer (am Vorbild Rilkes sich orientierenden) umfassenden existentiellen Verwandlung stellt Hans Egon Holthusen den Vorgang der Heimkehr aus dem Krieg:

Aber nun kehr ich zmack, getrankt mit dem Wesen

[der Toten,

Leise erheitert vom Weine des Übergangs, von der geheimen

Mitwisserschaft wie benommen, der inneren Übung des

Sterbens ${ }^{10}$.

11 - Nur in Ausnahmefällen erhält die Darstellung der Heimkehr im Gedicht einen unmittelbar politischen Zeitbezug, wie in Gedichten Bechers, in denen das Ich mit seiner Heimkehr die Hoffnung auf ein neues, verwandeltes Deutschland verbindet ${ }^{11}$;

12 - Weitaus häufiger knüpfen sich die Hoffnungsgefühle der Heimkehrenden an die Idee einer von Krieg und Zerstörung unberührt gebliebenen und in ihrer Lebenskraft ungeschwächten Natur: so z.B. bei Günter Eich, dessen «Wiederkehr»-Gedicht schon im Titel mit dem Doppelsinn von Rückkehr und Wiederholung spielt und die Heimkehr als Wiedereintritt in den alten Kreislauf des natürlichen Lebens darstellt:

Jenes rote Herbstgewölbe,

sommerliches Krötenloch:

Seht der Schimmer ist derselbe

aus dem Dickicht noch! ${ }^{12}$

13 Wie eh und je übernimmt die Natur hier die Funktion eines intakt gebliebenen Lebensraums, in dem der geschundene Mensch tröstliche Aufnahme und Kraft für neues Leben findet.

\section{II}

14 Auch die Lyrik Peter Huchels ist seit ihren Aufängen in den späten $20 \mathrm{er}$, frühen $30 \mathrm{er}$ Jahren durch eine sehr enge Beziehung zur Natur gekennzeichnet. Ihn in die Reihe der Naturlyriker um Wilhelm Lehmann zu stellen, fällt dennoch schwer. Zwar nehmen Naturbilder in seinen Gedichten eine dominierende Stellung ein, doch verbinden sich 
mit ihnen komplexe Bebeutungsebenen, deren Dichte an die Verschlüsselung und Chiffrenhaftigkeit hermetischer Lyrik erinnert. Neuere Untersuchungen, vor allem die Axel Viereggs, haben nachgewiesen, daß Huchels Lyrik in ihrer poetischen Eigentümlichkeit erst dann voll erfaßt wird, wenn man den Anteil der verschiedenen semantischen Schichten an Huchels Zeichensprache - Vieregg unterscheidet eine politische, eine existentielle und eine natur-mythische Schicht - erkennt:

In diesem Mitschwingen ... der verschiedenen «Schich

ten» ... liegt die Faszination seines Gedichts ${ }^{13}$

Das Gedicht «Heimkehr» wurde zuerst 1948 als Abschlußgedicht des Bandes «Gedichte» veröffentlicht, eine überarbeitete Fassung folgte 1967 in dem Band «Die Sternenreuse» ${ }^{14}$. Beide Fassungen weichen im Wesentlichen der Aussage und des Aufbaus nicht voneinander $a b$, allerdings ist im Übergang von der ersten zur zweiten Fassung der für Huchel kennzeichnende Prozeß einer zunehmenden Verknappung und Verdichtung zu beobachten ${ }^{15}$; die besondere Form der poetischen Bewältigung der Heimkehr-Situation ist in der ersten Fassung noch deutlieher zu erkennen.

Der Aufbau des Gedichts spiegelt eine Gedankenbewegung, deren einzelne Schritte genau mit der äußeren Gliederung zusammenfallen. - Zunächst benennt das Gedicht das Faktum der Heimkehr und gibt ihm eine raum-zeitliche Konkretisation aus dem Bliekwinkel des lyrischen Ich:

In der schwindenden Sichel des Mondes

kehrte ich heim und sah das Dorf,

verödete Hauser und Ratten.

Darauf folgt, als Signal einer Wendung des Blicks von der Wahrnehmung der äußeren Wirklichkeit zur geistigen Durehdringung und Verarbeitung des Wahrgenommenen, eine alleinstehende, mit einem Doppelpunkt abgeschlossene Zeile:

Über die Asche gebeugt, brannte mein Herz:

Der damit eröffnete Raum innerer Gedankentätigkeit wird in dem folgenden längeren und rhetorisch weit ausholenden Absehnitt mit Fragen ausgefüllt, die sich alle als Variationen einund derselben verzweifelten Frage nach dem Sinn eigenen Tuns angesichts der zerstörten Heimat zu erkennen geben:

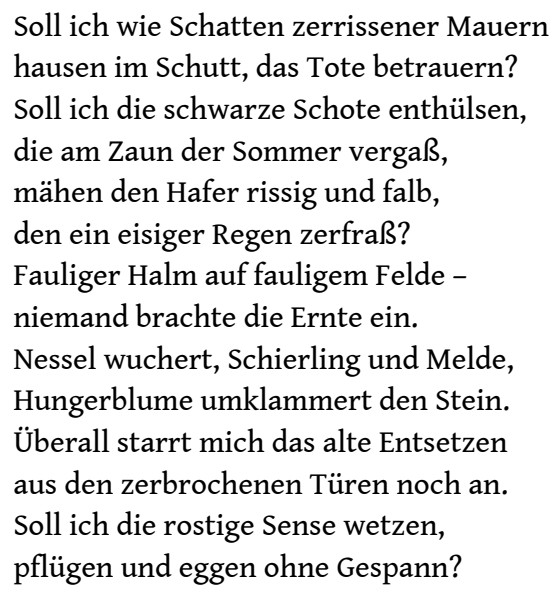

Nachdem auf diese Weise die Haltung des Ichs in ihrer ganzen Trostlosigkeit und Auswegslosigkeit vergegenwärtigt wurde, entwirft das Gedicht in den beiden letzten Abschnitten das Gegenbild einer hoffnungsvollen, zuversichtlich-tätigen Perspektive. Der Blick wendet sich dabei wieder nach außen und erfaßt einen scheinbar real stattfindenden Vorgang: 


\author{
Aber am Morgen, \\ es dämmerte kalt, \\ als noch der Reif \\ die Quelle des Lichts überfror, \\ kam eine Frau aus wendischem Wald. \\ Suchend das Vieh, das dürre, \\ das sich im Dickicht verlor, \\ ging sie den rissigen Pfad. \\ Sah sie schon Schwalbe und Saat? \\ Hämmernd schlug sie den Rost vom Pflug.
}

Dieser Vorgang besitzt zwar die voile sinnliche Anschaulichkeit eines wirklich gegenwärtigen Ereignisses, doch zugleich sind in ihm Momente enthalten, die ihn in ein seltsam unwirkliches, phantastisches Licht rücken. Und daß es sich bei dem Auftritt der «Frau aus wendischem Wald» um eine «Erscheinung» im doppelten Sinn des Wortes handelt, eher um ein Traum- und Phantasiebild als eine Wahrnehmung, bestätigt der letzte Abschnitt des Gedichts: in ihm wird die Frau aus ihrer konkreten Individualität («eine Frau ...») in die abstrakte Allgemeingültigkeit einer mythischen Gestalt überführt («Da war es die Mutter der Frühe ... die Muller der Völker») und so dem Ganzen der Charakter einer mythisch-utopischen Vision verliehen:

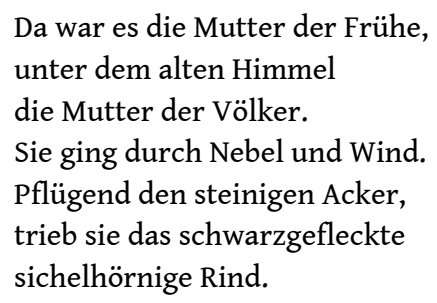

Aufs Ganze gesehen, bildet eine durchgehende Antithese das Grundmuster des Gedichts: auf der einen Seite die Hoffnungslosigkeit und Verzweiflung des Heimkehrenden, auf der anderen Seite der vertrauensvoll-tätige Neubeginn, repräsentiert durch die «Frau aus wendischem Wald», die als mythisch überhöhte «Mutter der Frühe» das Vieh zurückholt, den Rost vom Pflug hämmert und sich anschickt, von neuem den Acker zu pflügen und das Rind zu treiben. Die wesentliche Voraussetzung für Huchels Deutung der Heimkehr bildet hierbet die Vorstellung der «Mutter der Frühe», jener von Bachofen dem modernen Bewußtsein neu erschlossene Mythos weiblich-mütterlicher Begründung und Erhallung des Lebens in Gestalt der "Großen Mutter» ${ }^{16}$. Formal schlägt sich die Bedeutung der Antithese von Zerstörung und Neubeginn in einer Reihe von motivischen und syntaktischen Korrespondenzen zwischen den beiden Teilen des Gedichts nieder: so entspricht der «Heimkehr» des Ichs das «Kommen» der Frau («kehrte ich heim» - «kam eine Frau»), und dem Versinken des Ichs in grübelnder Untätigkeit das fraglose Tätigwerden der Frau, der zeitlichkosmischen Situierung der Heimkehr im Zeichen der «schwindenden Sichel des Mondes» die gleichfalls zeitlich-kosmische Situierung der Ankunft der Frau («am Morgen», «unter dem alten Himmel») usw. Vor allem aber, und dies erfordert ein näheres Eingehen auf die Funktionsbedingungen der Huchelschen Zeichensprache, sind der Anfang und das Ende, der erste, die Verzweiflung und Hoffnungslosigkeit darstellende, und der zweite, die Hoffnung und den Neubeginn darstellende Teil durch das Bild der «Sichel» miteinander verknüpft («In der schwindenden Sichel des Mondes» - «trieb sie das schwarzgefleckte / sichel hörnige Rind»), dem zudem am Ende des ersten Teils das Bild der «rostigen Sense» korrespondiert - eine strukturell 
hervorgehobene Korrespondenz, die bei Huchels erwiesener Sorgfalt im Umgang mit seinen Bildzeichen alles andere als zufällig oder belanglos ist.

III Ende $\mathrm{zu}$ der schon festgestellten antithetischen Bauform eine kreisförmige Strukturlinie hinzu. Es scheint so, als liege über der Darstellung von Anfang an ein versöhnend-utopisches Licht, als sei die Erneuerung des Lebens, in die das Gedicht die Heimkehr in die zerstörte Heimat münden läßt, als Verheißung und Hoffnung bereits in den ersten Worten verschlüsselt enthalten und als gehe die Darstellung von einer geheimen wechseiseitigen Beziehung zwischen dem Irdischen und dem Himmlischen aus, welche das Vertrauen in eine mögliche Regeneration menschlicher Lebenskultur rechtfertigt ${ }^{17}$. Möglicherweise ist dabei auch ein persönliches Moment im Spiel: der am 3. April, im Sternzeichen des Widders geborene Huchel hat wiederholt den Widder als Chiffre seiner eigenen Existenz in seine Gedichte aufgenommen ${ }^{18}$, so in dem 1973 entstandenen Gedicht «Der Ammoniter», in dem es (weitaus weniger hoffnungsfroh als in den früheren Gedichten) über die Beziehung zwischen Ich, Erde und Himmel heßt:

\footnotetext{
Mich verließen die alten Begleiter,

das Gleichgewicht von Erde und Himmel,

nur der Widder, die Moderhinke

schleifend über die Sterne, blieb mir treu.

Unter seinem Gehörn aus Stein,

das rauchlos glänzte, schlief ich nachts

usw $^{19}$.
}

Wie dem auch sei, die Sichel ist zweifellos ein von Huchel bewußt eingesetztes Bildzeichen, dessen Funktion sich nicht darin erschöpft, einen nur ornamentalen Verweis auf jene bäuerliche Sphäre der Mark Brandenburg, der Huchel entstammt, zu geben. Wie schon in dieser Sphäre - man vergleiche die Rolle, die die Sichel im Volksaberglauben und im bäuerlich-magischen Denken spielt ${ }^{20}-$, so nimmt auch in Huchels lyrischem Zeichensystem die Sichel eine bevorzugte Stellung als paradigmatisches Arbeitsgerät des Menschen im Umgang mit der Natur ein. Wenn in unserem Gedicht «Heimkehr» die Hoffnung auf eine Erneuerung des Lebens nach der Zerstörung bereits in der das Gedicht umspannenden Korrespondenz zwischen «Mond» und «Frühe» angelegt ist, so erhält diese Hoffnung erst durch das hinzutretende Zeichen der Sichel ihre spezifisch menschliche Fundierung ${ }^{21}$. In den «Notizen bei der Niederschrift einer Chronik, die das Gesetz der Bodenreform zum Inhalt hat», also zu jenem Versuch Huchels, die 1949 in der DDR durchgeführte Bodenreform in einem Lehrgedicht mit dem Titel «Das Gesetz» poetisch zu gestalten (der Versuch blieb Fragment), schreibt Huchel 1953:

Wenn sich der Dichter mit der Sprache der Arbeit, der Arbeitsgeräte, d.h. mit der Sprache des Volks beschäftigt ... so wird er im Gedicht ganz neue Wege gehen können.

Und weiter:

«Das Oesetz» ist das Leben selbst. Es wird sichtbar in jedem Feuer der Schmiede, in jeder Sense, jeder Hacke ... Es ist der tragende Grund für alles, was geschieht, indem es den Traum von Jahrhunderten wirklich macht ${ }^{22}$. 
Nimmt man diesen zeitgeschichtlichen und politischen Hintergrund hinzu, so wird der emphatische Sinngehalt, den Huchel dem «Sichel»-Bild gibt, verständlicher: gestützt auf die eigene Lebenserfahrung (den bäuerlichen Lebensbereich) und auf ein mythisches Lebens- und Naturdenken, in dem der Mensch seinen Platz als aktiver und produktiver Teil der Schöpfung einnimmt, gestaltet Huchel im Bild der «Sichel» (bzw. der «Sense» oder auch «Hacke», s.u.) die Utopie einer friedlichen Lebenskultur ${ }^{23}$. So kann die Sichel, wie in dem Gedicht «Mittag», in pathetisch-suggestiver Reihung ${ }^{24}$, oder, wie in dem Gedicht «Zunehmender Mond», mit dem idealen Anspruch des alten Regenbogen-Bildes als Zeichen der Versöhnung zwischen Himmel und Erde, Menschlichem und Göttlichem, in Erscheinung treten:

schöne Sichel,

Kommst geflogen,

Himmelsbrücke

hell gebogen ${ }^{25}$

Allerdings geht Huchels kosmischem Harmonie- und Gleichgewichts-Denken jede transzendent-religiöse Überhöhung ab. Die Basis bildet allein ein vertiefter Lebens- und Fruchtbarkeitsbegriff. In seinem Licht kann das Sichel- oder Sensen-Bild auch die Funktion eines phallischen Zeichens annehmen ${ }^{26}$. Das Moment der Gewalt, das mit ihm verbunden ist, kann dabei auch ins Zerstörerische umschlagen, wie Huchels Naturauffassung ja überhaupt frei ist von sentimentalen oder idyllischen Zügen ${ }^{27}$. In demselben Malße, in dem Huchels Vorstellung vom Mythos der «Großen Mutter» (s.o.) ambivalent ist und mit den lebensspendenden die zerstörenden und tödlichen GorgoAspekte verknüpft ${ }^{28}$, ist auch das Sichel-Bild ambivalent und kennt neben der befruchtenden und kultivierenden auch die zerstörerische Wirkung ${ }^{29}$. Dabei kommt es zu Anklängen an die verwandten traditionellen Vorstellungen von der «Hippe des Todes $»^{30}$ und von der «Sense» des Zeit-Gottes Saturn bzw. Kronos / Chronos. Mit ihr beginnt ein Gedicht, das derselben Entstehungszeit angehört wie das Gedicht «Heimkehr», «Die Pappeln»:

Zeit mit rostiger Sense,

Spät erst zogest du fort,

Den Hohlweg hinauf

Und an den beiden Pappeln vorbei ${ }^{31}$

In seiner ersten, Johannes R. Becher (dem damaligen Kultusminister der DDR) zum sechzigsten Geburtstag am 22.5.1951 gewidmeten Fassung trug das Gedicht noch den Titel «In der Heimat», und wie das «Heimkehr»-Gedicht thematisiert es zusammen mit der Erfahrung des Kriegs und seiner Zerstörungen die Hoffnung auf einen Neubeginn im Zeichen eines utopischmythischen Naturbegriffs. Auch in ihm dominiert die aus «Heimkehr» bekannte Korrespondenz zwischen dem Anfang und dem Ende des Gedichts, und war es in «Heimkehr» das Sichel-Zeichen, das diese Korrespondenz trug, so ist es hier das «glühende Eisen der Morgenröte», das dem Anfangsbild der «rostigen Sense» antwortet und ihm als dem geschichtlichen Zeichen der Zerstörung das hoffnungsvolle Zeichen menschlichkosmischer fruchtbarer Kultur gegenüberstellt:

Schon ist die Heimat,

Wenn über der grünen Messingscheibe

Des Teichs der Kranich schreit

Und das Gold sich häuft

Im blauen Oktobergewölbe;

Wenn Korn und Milch in der Kammer schlafen.

Sprühen die Funken 
Vom Ambo $\beta$ der Nacht.

Die rußige Schmiede des Alls

Beginnt ihr Feuer zu schüren.

Sie schmiedet

Das glühende Eisen der Morgenröte.

Und Asche fällt

Auf den Schatten der Fledermäuse ${ }^{32}$.

Beide Gedichte legen bei der am Anfang und am Ende berufenen Wechselbeziehung zwischen Erde und Himmel den Akzent auf das Moment menschlich-geschichtlicher, kultivierender Tätigkeit; in dem Titelgedicht des Bandes «Die Sternenreuse» erscheint dagegen das Verhältnis zwischen Mensch und All als ein eher passiv-vegetatives Einssein des Menschen mit der Natur, wie es sich dem rückblickenden Ich in der Erinnerung an seine Kindheit darstellt - das Gedicht ist ja auch eher ein Erinnerungsund Kindheitsgedicht als ein Zeitgedicht mit unmittelbar politischem Bezug. Bezeichnenderweise rückt in diesem Zusammenhang der Mond nicht als «Sichel», sondern als «Scheibe» in den Blick:

Daß du noch schwebst. uralter Mond?

Als jung noch deine Scheibe schwebte,

hab ich an einem Fluß gewohnt,

wo nur das Wasser mit mir lebte.

usw $^{33}$.

Auf diese Weise wird noch einmal der besondere Bedeutungsaspekt des Sichel-Zeichens als Chiffre einer paradigmatischen, auf friedliches Zusammenwirken von Mensch und Natur zielenden aktiven Teilhabe des Menschen an der Schöpfung sichtbar.

\section{IV}

Die Akzentverschiebung in der Darstellung des Mondes spiegelt sehr genau die Wandlung, die Huchels Lyrik vom scheinbar naiven Naturgedicht zum Zeitgedicht durchmißt Wo Huchel in seiner Lyrik auf zeitgeschichtliche Erfahrung reagiert, und er tut dies nach 1945 mit zunehmender Intensität, bildet ein mythischutopischer NaturBegriff die Folie, auf der eine Bewältigung der sich stellenden Probleme und eine Ausdrucksmöglichkeit der eigenen Erfahrung gesucht wird. Huchels lyrische Sprache nimmt die zeitgeschichtlichen Inhalte in sich auf und integriert sie so im Gedicht, da $\beta$ dessen poetische Gestalt zum Maßstab wird, an dem die Realität gemessen wird. Huchels Gedichte erfüllen so die Forderung Brechts, Spiegel im doppelten Sinne des Wortes zu sein ${ }^{34}$. Auf das Bild der Sichel angewandt: jene «Zeit mil rostiger Sense», mit der das Gedicht «Die Pappeln» einsetzt, ist keine geschichtslos-mythische Zeit, aber auch keine historischfixierbare Zeit allein, sondern beides in einem. Und so wird man auch davon ausgehen dürfen, da $\beta$ die politische Bedeutung, die dem Sichel-Zeichen in einer begrenzten zeitgeschichtlichen Perspektive zukommt, nämlich als politisches Emblem in Form des (auf Lenin zurückgehenden) ${ }^{35}$ «Hammer-und-Sichel» Zeichens, in Huchels Zeichensystem gleichfalls mitschwingt. Die Hoffnung, die das Gedicht «Heimkehr» am Bild der Sichel als utopisches Gegenbild zu den Zerstörungen des Kriegs entwirft, schließt die aktuelle zeitgeschichtliche Hoffnung auf einen neuen Beginn im DDR-Staat ein.

31 In dem Maße, in dem Huchels Gedicht sich als politisches versteht, wird es aber auch zum stillen, unerbittlichen Kritiker der zeitgeschichtlichen Entwicklung. Das Bild des 
gefesselten Widders im Eingangsgedicht des Bandes «Chausseen Chausseen», der 1963 nach der Entlassung Huchels als Chefredakteur der Zeitschrift «Sinn und Form» und dem Beginn der jahrelangen Isolation und Verfolgung durch den Sicherheitsapparat der DDR im Westen herauskam, dokumentiert die inzwischen erfolgte Wende: die politischen Hoffnungen wurden enttäuscht, der Neubeginn fand nicht statt, an die Stelle der Vision vom lebendigen Zusammenwirken zwischen Mensch und Natur tritt das Schreckbild einer sich selbst unterdrückenden und vernichtenden Menschheit:

Und nicht erforscht wird werden

Ein Geschlecht,

Eifrig bemüht,

Sich zu vernichten ${ }^{36}$

Dementsprechend verändert das Sichel-Zeichen seine Bedeutung. Erschien in dem Weihnachtsgedicht des Jahres 1953 «Dezembergang» die «Hacke» noch als Zeichen friedlichfruchtbarer Tätigkeit des Menschen:

Das Jahr, es gab uns Brot, nicht Steine,

Es gab dem Volk das ganze Korn.

Es schlug im heißen Julischeine

Die Hacke in den Disteldorn ${ }^{37}$

so wird sie nun zum Symbol der Unterdrückung durch die Staatsgewalt:

der Staat die Hacke,

das Volk die Distel ${ }^{38}$

Entsprechend ändert sich aber auch die Stellung des Ichs. Seine utopisch-mythische Position «unter der schwindenden Sichel des Mondes» wird brüchig, das Vertrauen in eine wiederherstellbare Harmonie zwischen Mensch und Schöpfung zerfällt, an ihre Stelle tritt die neue, für Hoffnung keinen Raum bietende Positionsbestimmung «unter der blanken Hacke des Monds»:

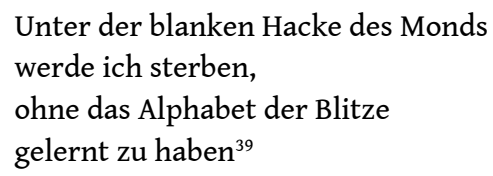

Jene Möglichkeit der «Heimkehr», die im Jahr 1948 noch als utopischer Entwurf ans Ende eines Gedichtbandes gestellt werden konnte, hat sich einmal mehr als Täuschung erwiesen. Was bleibt, ist die im Gedicht aufbewahrte Hoffnung, daß einmal dennoch «wirklich» werde, was vorerst als «unwirklich» und irreal gelten muß:

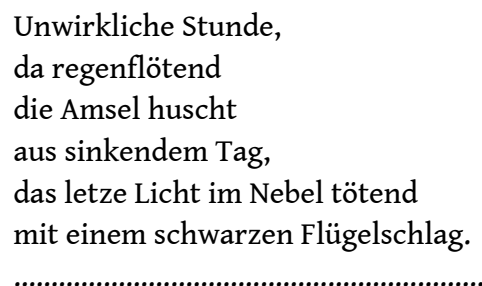

O wirkliche Stunde, da laubkühl flötend die Amsel huscht in werdenden Tag, die eisengraue Frühe rötend mit einem leisen Flügelschlag ${ }^{40}$. 


\section{NOTES}

1. «Die Heimat», in: Hölderlin, Sämtliche Werke, hg. von Fr. Beißner (Kleine Stuttgarter Ausgabe), Bd. 2, Stuttgart 1961, S.19.

2. «Heimkehr», in: Franz Kafka, Sämtliche Erzählungen, hg. von P. Raabe, Frankfurt 1 M. 1970, S. $320 \mathrm{f}$.

3. «Heirnkehr», in: Nachkrieg und Unfrieden. Gedichte als Index 1945-1970, hg. von H. Domin, Neuwied, 1970, S. 22.

4. Johannes R. Becher, Werke in 3 Bänden, Berlin (Ost) 1976, Bd. 1, S. 400.

5. In: Nachkrieg und Unfrieden, S. $10 \mathrm{f}$.

6. «Ich kehrte heim», in: «Ich sah aus Deutschlands Asche keinen Phönix steigen». Rückkehr und Hoffnung in poetischen Zeugnissen, hg. von B. Jentzsch, München 1979, S. 126 f.

7. Ricarda Huch: «Braunschweig», in: «Ich sah aus Deutschlands Asche keinen Phönix steigen», S. 29; Marie Luise Kaschnitz: «Rückkehr nach Frankfurt», ebda S. $30 \mathrm{ff}$.

8. In: Bertolt Brecht, Gesammelte Werke in 8 Bänden, Frankfurt/M., 1967, Bd. 4, S. 876, 858.

9. «Frühjahr 1946», in: E.L., Mithras. Lyrik und Prosa, hg. von O.F. Best, Frankfurt/M., 1959, S. 31.

10. «Heimkehr», in: H.E.H., Hier in der Zeit. Gedichte, München 1949, S. 32 ff.

11. Vgl. «Heimweg», in : Werke Bd. 1. S. 400; «Abschied und Wiederkehr», in: Werke Bd. 1, S. 400.

12. In: G.E., Abgelegene Gehöfte. Gedichte, Frankfurt/M. 1968, S. 84.

13. Axel Vieregg, Die Lyrik Peter Huchels. Zeichensprache und Privatmythologie, Berlin 1976, S. 146.

14. In: Peter Huchel, Gesammelte Werke in 2 Bänden, hg. von A. Vieregg, Frankfurt/M., 1984 (künftig abgekürzt: GW), Bd. 1, S. 109f. und Bd. 1, S. 171.

15. Vgl. Vieregg a.a.O. S. 39 (zu «Hinter den weißen Netzen des Mittags») und Hubert Ohl, Peter Huchel: Das lyrische Werk im Spiegel seiner Titelgedichte, in: Literatur in Wissenschaft und Unterricht 16 (1983), S. 281-300 (zu den Fassungen von «Gezählte Tage», S. 294 ff.).

16. Hierzu: Ludvik Kundera, Die wendische Mutter, in: Über Peter Huchel, hg. von H. Mayer, Frankfurt/M. 1973, S. 111-118; Vieregg a.a.O. $91 \mathrm{ff.}$

17. Zur Idee des Gleichgewichts zwischen Erde und Himmel vgl. Vieregg a.a.O. S. 126f.; Ohl a.a.O. S. 288.

18. Vgl. Vieregg a.a.O. S. 133.

19. GW 1, 230; zur Deutung des Gedichts: A. Vieregg, Ein Gedicht nach Auschwitz. Peter Huchels «Der Ammoniter», in: Festschrift for E.W. Herd, hg. von A. Obermayer, Dunedin 1980, S. 263-274.

20. Vgl. hierzu: Handwörterbuch des deutschen Aberglaubens, hg. von H. Bächtold-Stäubli, Berlin 1927 ff. (u.a. ist für die Mark Brandenburg bezeugt der Brauch, zum Schutz des Viehs vor Hexen eine Sense oder Sichel ins Futter zu legen: Bd. 6, Sp. 236).

21. Vieregg hebt in seinen Ausführungen zu dem «Heimkehr» - Gedicht (a.a.O.S. 91 ff., besonders S. 100 ff.) die strukturelle Funktion der «Sichel»-Korrespondenz hervor, geht aber nicht weiter auf die Bedeutung des «Sichel»-Zeichens in Huchels Bildsprache ein.

22. W 2, $293 \mathrm{f}$.

23. Diese Bedeutung legt Huchels Gebrauch der «Sichel» (bzw, der «Sense») in folgenden Gedichten nahe: «November» (GW 1, 205), «Im Kun-Lun-Gebirge» (GW 1, 256), «Unterwegs» (GW 1, 246), «Das Gesetz» (GW 1, 290), «Die Schwalbe» (GW 1, 100), «Schlucht bei Baltschik» (GW 1, 125), «Lenin in Rasliw» (GW 1, 300), «Ankunft» (GW 1, 177).

24. GW $1,127$.

25. GW 1, 92; in der ersten Fassung noch expliziter: «Erd' und Himmel/Will sich mischen» (GW 1, 279; zur Deutung vgl. Vieregg a.a.O. S. 104).

26. Vgl. das Gedicht «Der polnische Schnitter» (GW 1, 54; zur Deutung Vieregg a.a.O, S. 116 ff). 
27. In einem Rundfunk-Interview äußerte Huchel 1974: «Ich habe eine Kindheit auf dem Lande verbracht, und die Natur war für mich nicht mehr die heile, die absolute Natur, sondern es war für mich die vom Menschen veränderte Natur, in der er leben konnte. Die Natur ist für mich etwas sehr Grausames... Die Natur war für mich Fressen und Gefressenwerden» (GW 2, 393).

28. Hierzu Vieregg a.a.O. S. $128 \mathrm{ff}$.

29. Vgl. Etwa «Die Sichel Satans» (GW 1, 32), «Exil» (GW 1, 178: «Der Sommer legt dir die eiserne Sichel aufs Herz»). «Münze aus Bir el Abbas» (GW 1, 130), sowie das Bild der Hacke in den Gedichten «Hubertusweg» (GW 1, 223) und «Unter der blanken Hacke des Monds» (GW 1, 211).

30. Vgl.: «Und stumpf wird so des Todes Hippe» («Dezembergang», GW 1, 296).

31. GW l, 145.

32. GW l, 146.

33. GW 1, 83.

34. Vgl. Brechts Bemerkung zu Wordsworths «She was a phantom of delight» in seinem Arbeitsjournal (24.8.1940): «der unterschied liegt zwischen "widerspiegeln" und "den spiegel vorhalten"» (B.B. Arbeitsjournal, hg. von W. Hecht, Frankfurt/M., 1974, S. 126).

35. Vgl. Gert Oswald, Lexikon der Heraldik, Mannheim 1984, S. 174.

36. GW 1,157 («Psalm»).

37. GW l, 296

38. GW 1, 223 («Hubertusweg») (= Name der Straße, in der Huchel in Potsdam-Wilhelmshorst wohnte).

39. GW 1, 211 («Unter der blanken Hacke des Monds»).

40. GW 1, 293/295 («Chronik des Dorfes Wendisch-Luch»).

\section{RÉSUMÉS}

Neben der das moderne Bewußtsein so sehr prägenden Erfahrung der «unmöglichen Heimkehr» kennt die deutsche Literatur des 20. Jahrhunderts auch Beispiele unmittelbar erfahrener Heimkehr: der Heimkehr aus Krieg und Zerstörung. An Huchels Gedicht Heimkehr wird untersucht, in welcher Weise diese Erfahrung ästhetisch verarbeitet und gestaltet wird. Vor dem Hintergrund der zeitgeschiehtlichen Situation einerseits, der poetischen und weltanschaulichen Voraussetzungen des Lyrikers Huchel andererseits, erscheint das Gedicht als ein utopischkonstruktivistischer Entwurf, dessen ideelle Kraft auch dann noch wirksam ist, wenn sich die an ihn geknüpfte reale politische Hoffnung als vergeblich erweist.

À l'expérience de l'impossible retour qui marque si profondément la conscience moderne, la littérature du vingtième siècle ajoute aussi des exemples de retours réellement vécus : au sortir de la guerre, de l'anéantissement. Le poème de Peler Huchel Heimkehr permet d'étudier une transposition littéraire de cette expérience. Etant donnés l'arrière-plan historique d'une part, les conceptions esthétiques et philosophiques de Huchel poète d'autre part, ce texte apparait être un projet d'utopie constructive : le pouvoir de ses idées reste opérant, même si l'authentique espoir politique qui va de pair avec ce projet se révèle vain. 
AUTEUR

LUDWIG VOLKER

Universität Münster 